\title{
Ejection mechanisms in the sublayer of a turbulent channel
}

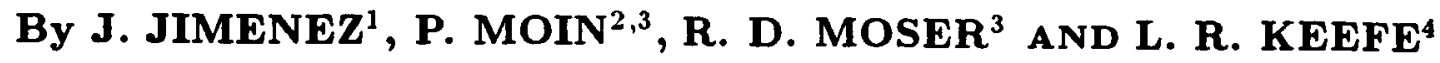

It has long been recognized that the structure of wall bounded turbulence in boundary layers and channels is three-dimensional (Kline et al., 1967). A generally accepted picture is that low velocity streaks are ejected from the wall layer and are responsible for a large fraction of turbulence production. (see e.g., Cantwell, 1981). The mechanism that triggers the initial ejection is, however, not understood, and there are indications that the processes controlling the behavior of the viscous sublayer, where these ejections originate, are different from those active in the outer parts of the boundary layer.

Recently, Jimenez (1987), while studying numerically the behavior of a two. dimensional channel flow, found a simple mechanism giving rise to spontaneous ejections of vorticity, and of the associated low momentum fluid, away from the wall and into the core flow. In that 2-D case, once the vorticity is ejected, it is stretched into long thin shear layers which periodically release part of their vorticity into the laminar core of the channel, where it is eventually dissipated by viscosity (figure 1). It should be stressed that the only vorticity component present in a 2-D flow is $\omega_{z}$, and that this process is essentially different from any of the usually accepted mechanisms involving induction by hairpin vortices, which include important contributions from $\omega_{x}$, and $\omega_{y}$. As such, it was not expected that this complete picture would survive in fully developed three-dimensional channel flows, but one of our goals during this workshop was to check whether some aspect of it could still be useful in describing fully turbulent situations.

A particularly appealing possibility was that the same mechanism could contribute to the origin of the ejections in natural channels, especially since it was shown in Jimenez (1987) that the site of the basic ejection instability for the 2-D flow is the viscous sublayer, where it can reasonably be expected to be approximately independent of the three-dimensional phenomena occurring in the outer part of natural boundary layers.

The general behavior of the 2-D solution is that of a periodic train of nonlinear Tollmien-Schlichting (T-S) waves, of the kind described in (Herbert, 1976). This wave train is characterized by a succession of strong vorticity peaks at the wall, separated by regions of weaker, or even negative vorticity. This alternation of strong and weak vorticity generates local updrafts, corresponding to stagnation points in

1 Universidad Politecnica, Madrid, and UAM-IBM Scientific Center, Spain.

2 Stanford University

3 NASA Ames

4 Center for Turbulence Research 

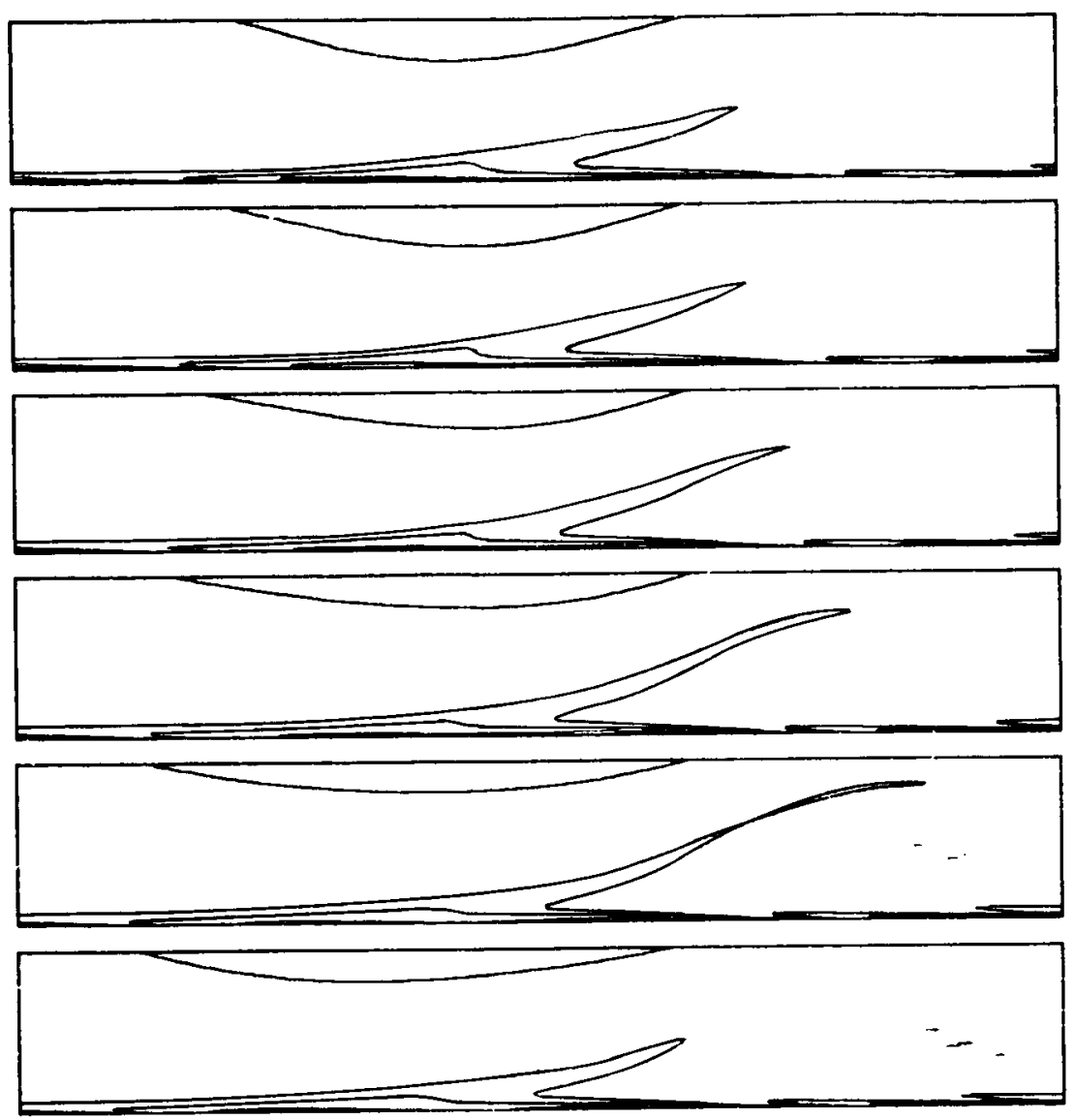

Figure 1. Two dimensional ejection in the boundary layer of a 2-D channel. The periodic ejections from the train of nonlinear Tollmien- Schlichting waves are equivalent to a limit cycle of the system (from Jimenez, 1987). Flow runs from left to right, and time, from top to bottom. Axes move with primary T-S wave. Each frames spans from the lower wall to the channel centerline.

a frame of reference moving with the wave train, which tend to draw vorticity away from the wall, producing the protruding shear layers (see figure 2). The stability of this situation depends on the Reynolds number. Above $R e=U h / \nu=5500$, and for a wave number $\alpha=1.0$, the uniform wave train becomes unstable and bifurcates into a limit cycle, giving rise to the periodic ejections described above. At a higher $R e=9100$, it bifurcates again into more complicated dynamical behavior (a torus). Here, $U$ refers to the center-line velocity of a parabolic profile with the same mass flux, and $h$, to the channel half width. Throughout this paper we will use this non-dimensionalization.

Our first step was to check the accuracy of the original 2-D calculations. To do that, some initial conditions from Jimenez (1987) were used with a 2-D version of the channel flow described in (Kim, Moin \& Moser, 1987). Although both numerical codes are spectral, they are essentially independent, and differ in many important 


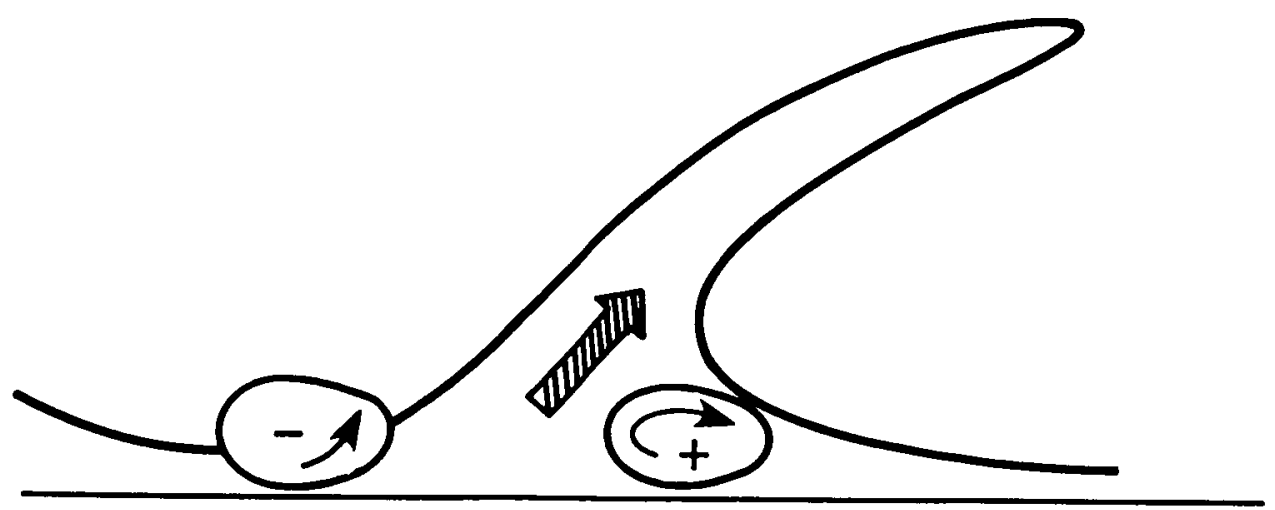

FIGURE 2. The basic mechanism for the two dimensional ejection of vorticity is the creation of an updraft between a pair of spanwise vortices of opposite sign (or unequal magnitude) in the sublayer.

details, including different dependent variables used in the integration. Nevertheless, the results of both codes did check in detail. The comparisons included a limit cycle $(R e=7000)$, and a torus $(R e=9200)$. In both cases, not only the qualitative nature of the results from both codes were similar, but the quantitative values of the wall stress, and of its oscillations as a result of the instability, agreed to within plotting accuracy.

The next step was to investigate the degree of similarity between nonlinear T-S waves and thin layers of $z$-vorticity present in natural channel flows. For that, we used a short time series of flow fields extracted from the numerical simulation described in (Kim, Moin \& Moser, 1987). This is a fully resolved numerical simulation, $R e=4200$, of a channel which is defined as $4 \pi h$ periodic in the $x$ direction, and as $4 \pi h / 3$ periodic in the $z$ direction. The Reynolds number is based on the centerline velocity of a parabolic profile with the same mass flux. It was shown in that reference that its statistical properties are in good agreement with those of experimental flows, and we will consider it here as a "natural" turbulent channel.

The first surprising observation is that thin layers of $z$-vorticity are indeed a very common feature of this channel flow, and that they protrude from the wall in a manner which is strongly reminiscent of the features observed in the 2-D calculations (figure 3). To our knowledge, this is a new observation, although Kim (1987) had described the formation of thin layers of high vorticity magnitude as part of the evolution of an isolated "hairpin" vortex in the neighborhood of a channel wall.

There are some important differences between the structures observed in the channel and those in the 2-D calculations. To begin with, the "wavelength" seems to be shorter, with an average longitudinal separation between consecutive features of the order of 1 to $3 h$. (200-600 wall units), while the 2-D nonlinear T-S waves can only exist, as equilibrium solutions, for wavelengths in a range between $4 h$ and $6.5 h$. Also, the channel layers penetrate less into the core of the channel, appearing to level off at a distance of $0.3 h$. ( 35 wall units) away from the wall, while the 2-D 


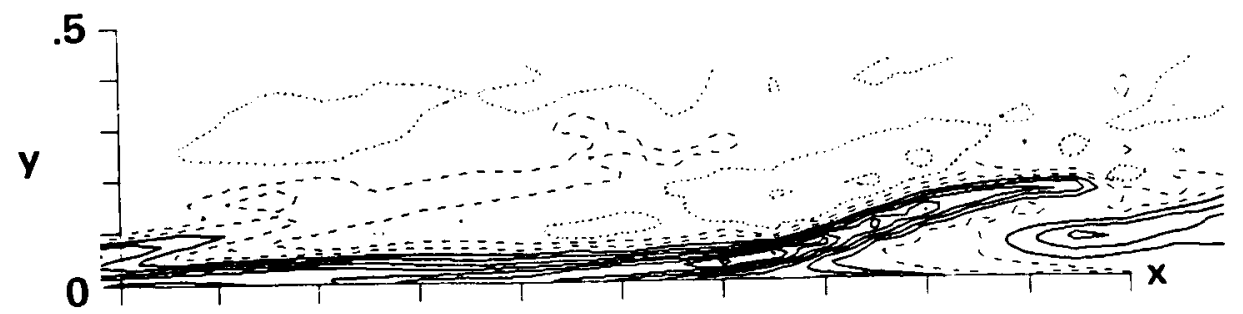

FIGURE 3. Lateral view of a train of projecting shear layers of $z$-vorticity in a natural, fully developed three dimensional channel flow. Note the similarity to the structures in the 2-D calculations. Dotted lines correspond to $\omega_{z}=-1,0$; dashed: $\omega_{z}=1,2$; solid: $\omega_{z}=3$ to 17 . Average vorticity at the wall is $\omega_{z}=7.67$.

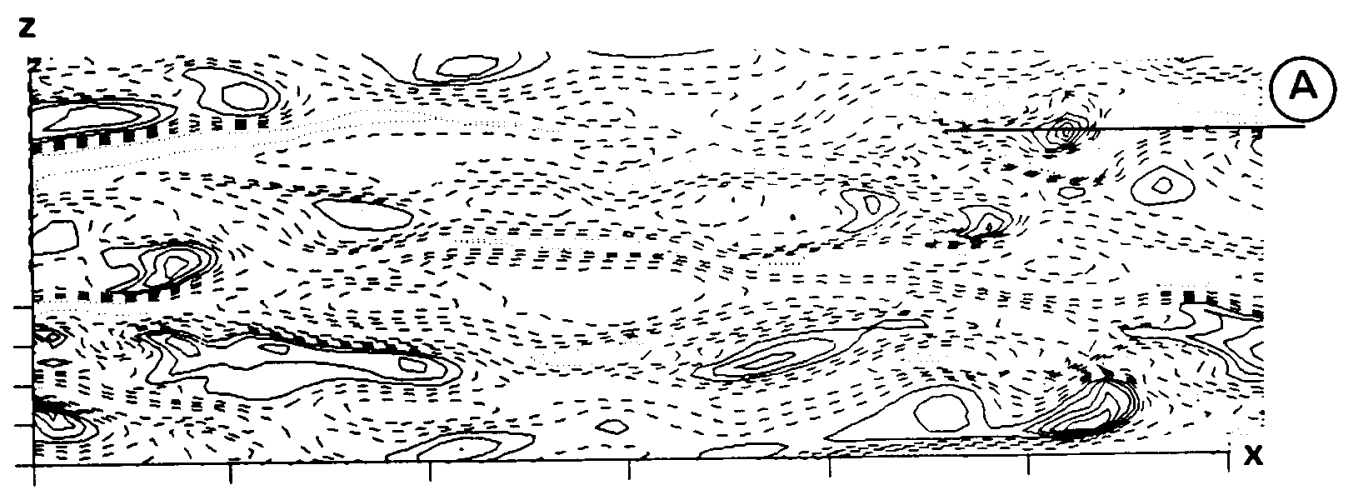

FIGURE 4. Distribution of spots of high spanwise vorticity in the viscous sublayer $\left(y^{+}=6\right)$. These spots are the roots of the structures in figure 3 . The line marked "A" corresponds to the position of the cross section in figure 3 .

solutions extend all the way to the channel center line. On the other hand, there is some evidence, in the natural flow, of weaker layers that do extend deeper into the core.

The main difference, however, is that the shear layers in the natural channel are three dimensional structures, with a spanwise extent of no more than $0.2 h$, or about 35 wall units, at $y^{+}=6$ (and about twice as much at the wall). They appear to be rooted at the wall in elliptical "hot" spots in which the spanwise vorticity is at least $25 \%$ higher than its average wall value, and to extend into the channel with a characteristic S-shape, and an average ejection angle of a few degrees. These spots can be used to detect and count the protruding layers and to follow their motion (figure 4). They appear to move with a convection velocity of $60 \%$ of the center line velocity, or $0.47 U$, where $U$ refers to the centerline velocity of the laminar profile at the same mass flux. This last number is in surprisingly good agreement with the convection velocity of the $2-\mathrm{D}$ nonlinear waves. Although the significance of this 


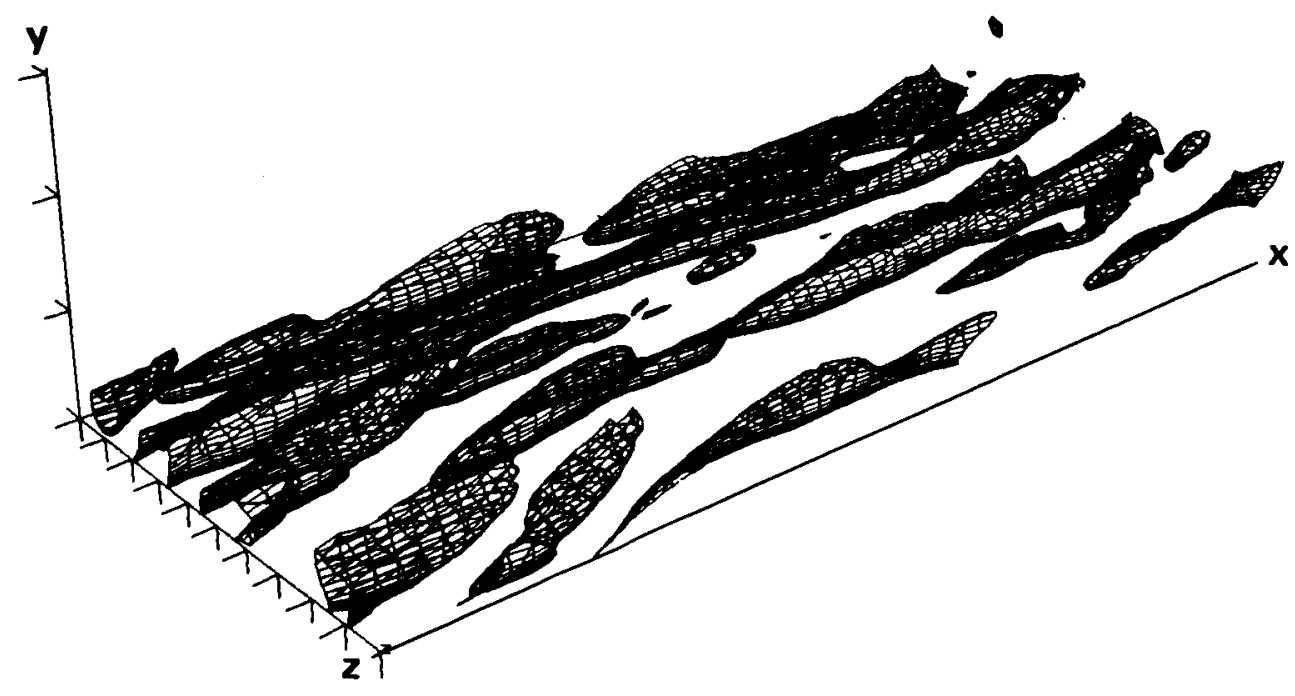

FIGURE 5. Three dimensional representation of $z$-vorticity structures extending away from the viscous sublayer. The vorticity iso-surface represented is approximately $25 \%$ higher than the average wall vorticity, and the first tick mark in the $y$-axis corresponds to $y^{+}=17$.

agreement is not clear, this convection velocity is quite high, showing that the spots are linked to some structure extending outside the viscous sublayer.

In fact, when these hot spots are followed into the flow in the form of three dimensional iso-surfaces of $z$-vorticity, they form a "forest" of leaning curving "necks" that covers much of the wall (figure 5). It is possible to follow the evolution of these structures as they move in time, and some of them were followed for fairly long periods, long enough for the structure to move several channel half widths. In the course of their life they reproduce, giving origin to new structures, and we were able to observe several of these reproduction processes. An example is given in figure 6 , where time runs from top to bottom. In the first frame of this time sequence a structure has began to stretch, producing a small vorticity blob at its top end. In the next frame the blob has grown considerably, and a small patch of strong vorticity appears at the wall. Finally the vortex at the walls grows out into the boundary layer and fuses with the tip of the stretching layer. At this moment, the tip separates from its parent structure, forming what appears to be the "embryo" of a new spot. The last frame shows both spots as essentially independent units.

A closer examination of the vorticity field shows that there is a region of concentrated $z$-vorticity of opposite sign (negative), underneath the top part of the structure. These regions of reverse vorticity are visible in the lateral view of this same structure in figure 3 . The whole reproduction process is strikingly reminiscent of the instability process for 2-D linear Tollmien-Schlichting waves (see Betchov \& Criminale, 1967). Basically, vorticity is created at the wall and diffuses into the main flow through viscosity. In a frame of reference moving with the structure, the fluid below the critical layer is moving backwards, while that on top moves forward. 


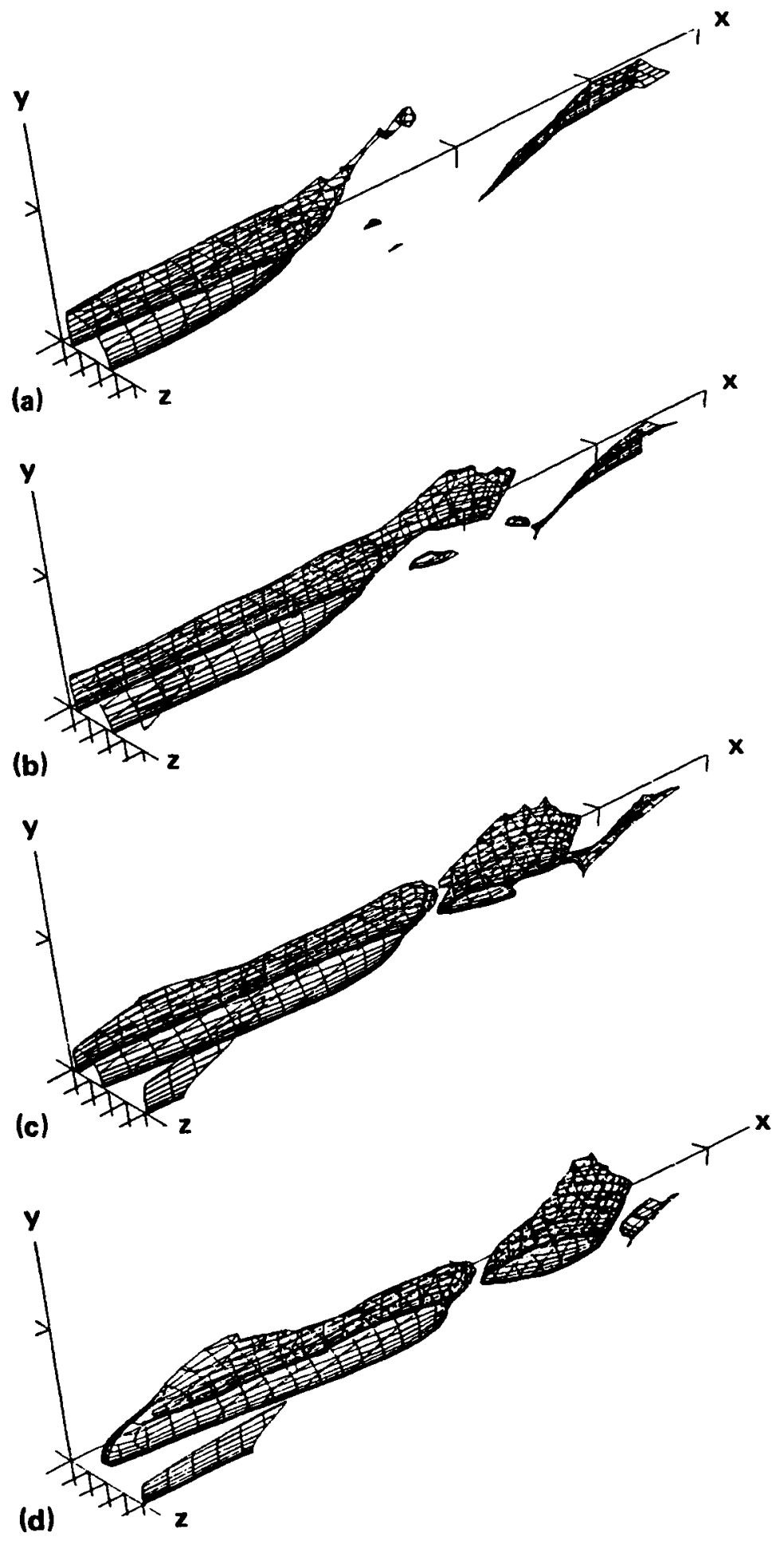

Figure 6. Splitting process of a structure to create a new one. See text for explanation. Time difference between frames is approximately 6 wall units $\left(\nu / u_{\tau}^{2}\right)$. 
As a result, as the vorticity diffuses away from the wall, it takes a backwards pointing " $V$ " shape that is visible in figure 3 . Eventually, the positive vorticity in the main structure (above the critical layer) induces a negative vortex near the wall, to accommodate the no slip condition. This vorticity of opposite sign is convected backwards by the flow, as it diffuses outward, and forms a negative vortex layer underneath the original positive one. This new layer eventually overcomes the effect on the wall of the original structure, and begins to induce a new positive vortex. The moment that a strong vortex pair in formed in this fashion, underneath the original structure, an updraft is created that carries part of the positive and negative vorticity into the upper part of the structure. The negative vorticity in the rear cuts the connection between the head and the base of old structure (through viscous annihilation), while the positive vorticity connects with the head of the old structure to form a new one (see figure 7 ). Note that the mechanism invoked here, for the production of the updraft, is the same one proposed in figure 2 for the ejection of shear layers into the flow.

As noted previously, this is the mechanism responsible for the linear 2-D T-S waves, and it seems to explain approximately the behavior of $z$-vorticity in the splitting mechanism in figure 6 . This suggests that the mechanism for the generation of ejections in the sublayer may be essentially two dimensional, although there are undoubtedly some three dimensional effects present, as shown by the fact that the structures do not spread laterally into spanwise bands. In fact a map of $y$-vorticity in the sublayer shows long active streaks, delimiting quiescent. "corridors" between them (figure 8). The hot spots, and their associated shear layers seem to ride those corridors, as a necklace of beads. $x$-vorticity is also present in the sublayer, but it seems to be weaker, and harder to correlate with the structures studied here. Also, there is little doubt that, as the shear layers are ejected further into the main stream, longitudinal vorticity and three dimensional effects are important in their evolution.

The general picture of the sublayer suggested by this simplified model is a collection of patches of the high vorticity in the wall layer, which are lifted into little "ramps" corresponding to the shear layers described in this paper. Since the vortex lines cannot end in the middle of the flow, these ramps are linked to the wall by "sidewalls" which correspond to the regions of high $y$-vorticity observed in figure 8 . It is easy to see that, if the ramps are constrained to be in between the streaks, the picture becomes something like the one in figure 9, and the induced longitudinal velocity fluctuations in the sublayer should consist of high velocity narrow streaks, bounding wider bands of lower velocity. This is precisely the pattern observed in experiments.

The remaining question is whether the three dimensional structure representing one of the protruding ramps can be studied in isolation. Numerical simulation provides a unique opportunity to attempt this, since the behavior of structures in natural channels is complicated by the interactions among the large number of them present in the flow, and since it is obviously difficult to isolate a single structure in a physical experiment. The numerical equivalent of isolating a single structure is 

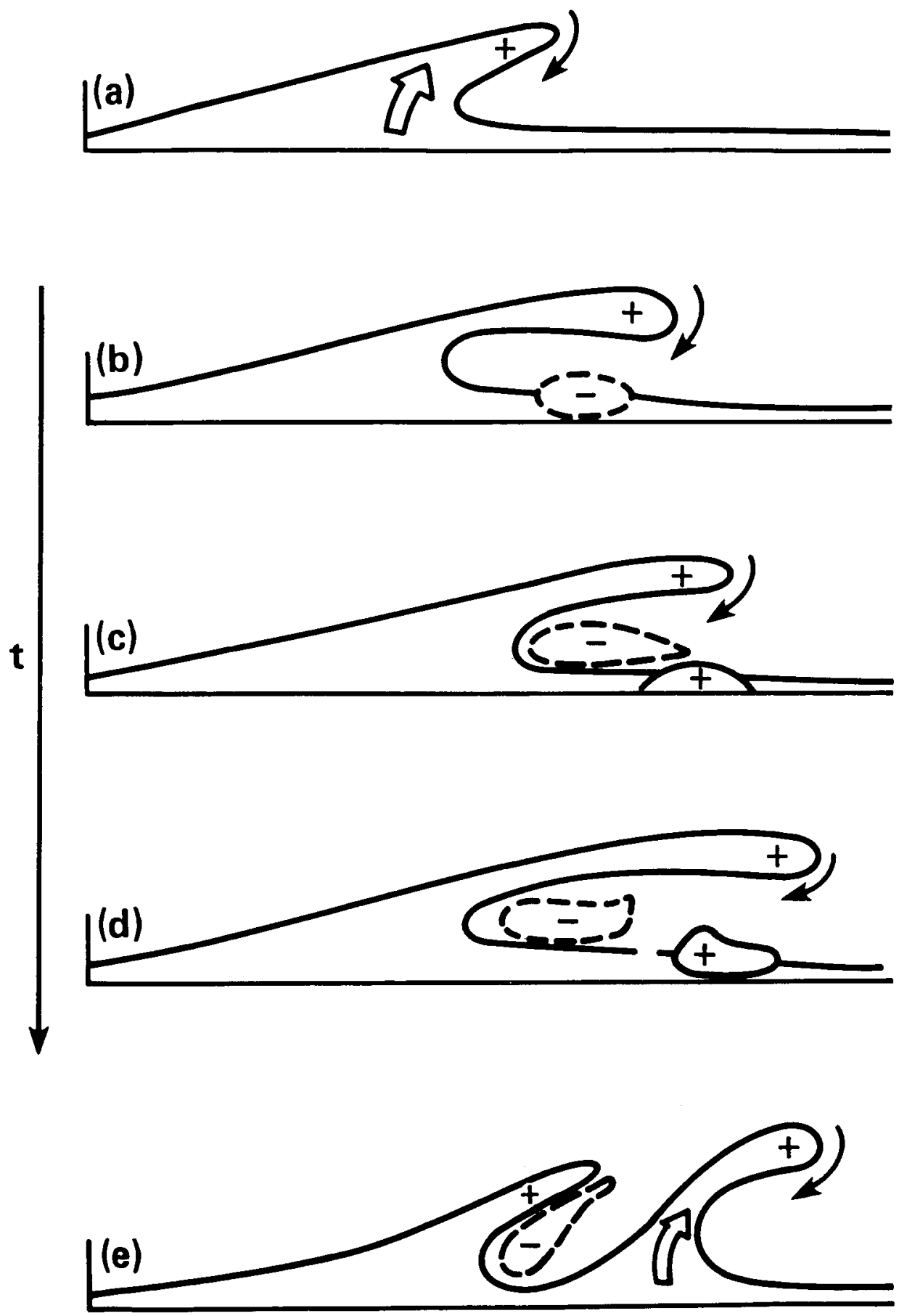

Figure 7. Tentative mechanism for the splitting process of a structure. This 2-D mechanism corresponds roughly to the instability mechanism for TollmienSchlichting waves. 
2

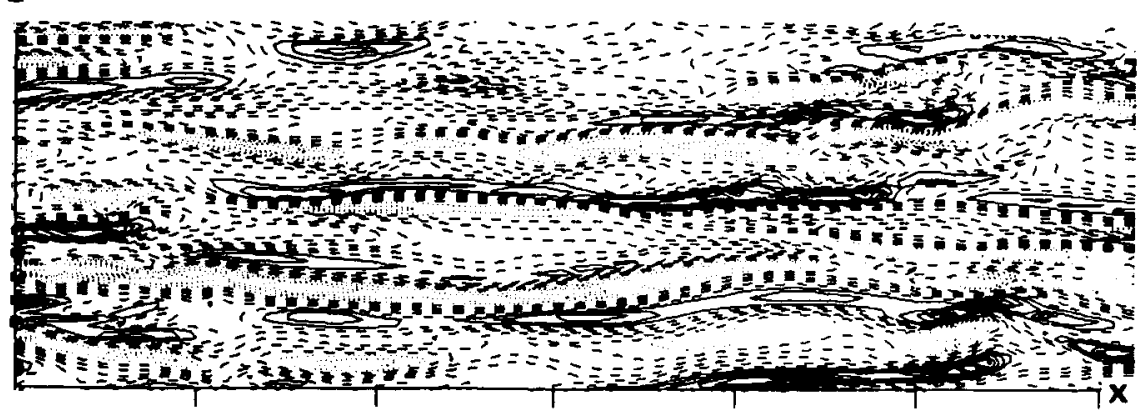

FIGURE 8. Streamwise streak pattern of $y$-vorticity in the sublayer. Conditions are identical to those of figure 4, and careful comparison with that figure shows that the vorticity spots reside in the relatively quiescent corridors between the high velocity streaks.

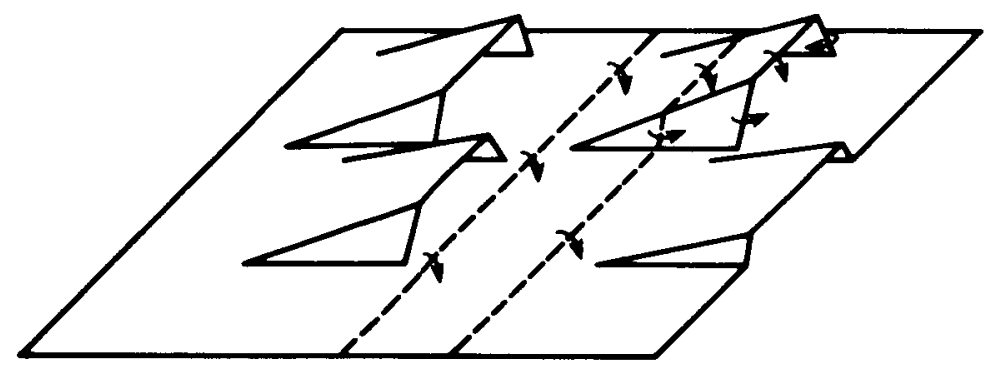

FIgURE 9. A model for the sublayer structures. The raised structures are lifted portions of wall vorticity, and are "supported" by vorticity sidewalls that are the origin of the $y$-vorticity streaks.

to use a computational box whose $x$ - $z$ extent is small enough to contain only one, or at most a few, structures. This was attempted in the course of the workshop.

Running at $R \epsilon=7000$, we first tried computing a channel on a periodic box of $2 \pi h \times 2 \pi h$ (about $1800 \times 1800$ wall units). The initial conditions were extracted from the 2-D limit cycle solution, with the addition of a small $3-D$ perturbation. As expected, the flow became quickly three-dimensional, and the wall shear stress grew from the low value corresponding to the 2-D (Herbert) solution $\left(\omega_{z}=3\right.$ ), to that for a fully developed turbulent channel $\left(\omega_{z}=12\right)$. This solution was not followed for a long time, since it was not any easier to understand than any of the previously available flow fields. The remaining numerical experiments were carried out using computational boxes whose spanwise extent was $\pi h / 8$, corresponding to approximately 113 wall units. Since the computational domain is periodic in both $x$ and $z$, this corresponds to a periodic array of structures with a spanwise spacing close to the one found in natural flows. We tried different streamwise 


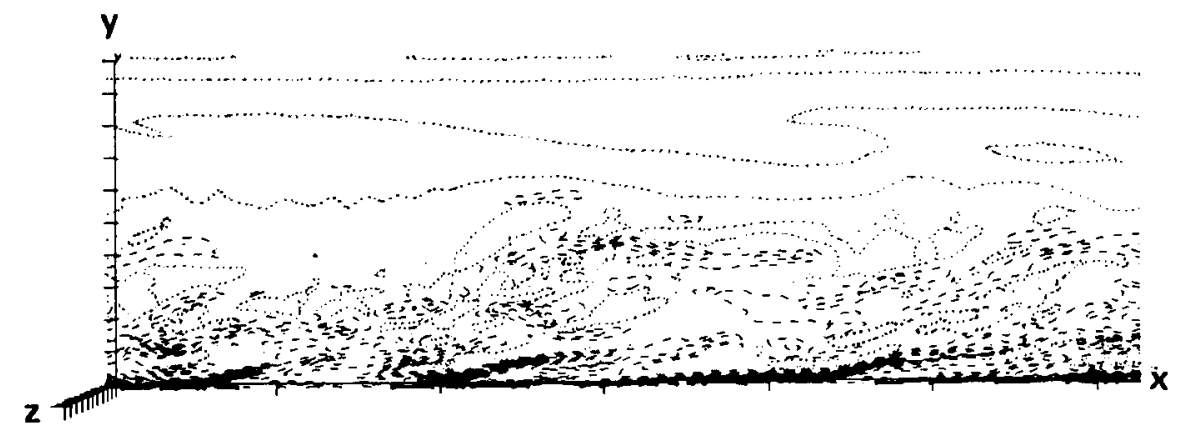

FIGURE 10. z-vorticity field of a 3 -D narrow channel, as described in the text. Note that the upper wall, at the top of the figure, is essentially two-dimensional, and smooth, while the lower one remains three-dimensional and "turbulent".

periods, none of which was completely successful. The most desirable boxes were the very short ones, that could be expected to contain a single structure (actually a doubly periodic array of them). However, attempts to introduce perturbations in boxes with streamwise periods of $\pi h / 8$, and $2 \pi h / 5$ quickly decayed to laminar solutions. The most interesting box, up to now, has been one with an $x$-extent of $2 \pi h$, and a spanwise one of $\pi h / 8$. This flow cannot decay to laminar, since it is linearly unstable to two dimensional perturbations of this wavelength. Apparently, however, that domain is neither sufficiently stable to decay to a $2-\mathrm{D}$ solution, nor sufficiently unstable to maintain a fully 3-D turbulent one. The result is a nonsymmetric channel in which one wall (the top one in our case) sustains a roughly 2-D flow, while the other one has a turbulent, 3-D boundary layer, presumably forced by the 2-D solution at the top wall (see figure 10). This boundary layer contains ejection structures similar to those in 3-D natural layers, and which appear to be fairly typical, but the average shear stress at the wall falls in between the values characteristic of $2-\mathrm{D}$ and $3-\mathrm{D}$ solutions. At each particular moment, the computational box contains 3 or 4 sublayer structures, which are still too many for a simple model of the layer, but constitute, at this moment, the smallest system available for its study. Further experiments with boxes of different sizes are still in progress.

\section{Summary}

In summary, we present here a possible model for the inception of vorticity ejections in the viscous sublayer of a turbulent rectangular channel. We have shown that this part of the flow is dominated by protruding strong shear layers of $z$ vorticity, and have proposed a mechanism for their maintenance and reproduction which is essentially equivalent to that responsible for the instability of 2-D TollmienSchlichting waves. The efforts to isolate computationally a single structure for its study have failed up to now, since it appears that single structures decay in the absence of external forcing, but a convenient computational model has been identified in the form of a long and narrow periodic computational box containing at each 
moment only a few structures. Further work in the identification of better reduced systems is in progress.

\section{REFERENCES}

Betchov, R. \& Criminale, W. 1967 Stability of parallel flows. Academic Press, Ch. II.

Cantwell, B.J. 1981 Organised motion in turbulent flow . Ann. Rev. Fluid Mech. 13, 457-515.

Herbert, T. 1976 Periodic secondary motions in a plane channel . Proc. 5th Int. Conf. Num. Methods in Fluid Dyn. (A.I. Van de Vooren \& P.J. Zandbergen, eds.). Springer, pp.235-240.

Jimenez, J. 1987 Bifurcations and bursting in two-dimensional Poiseuille flow. Phys. Fluids, in press.

KIM, J. 1987 Evolution of a vortical structure associated with the bursting event in channel flow. Turb. Shear Flow 6, Springer, pp. 221-233.

Kim, J., Moin, P. \& Moser, R. 1987 Turbulence statistics in fully developed channel flow at low Reynolds number. J.Fluid Mech. 177, 133-166.

Kline, S.J., Reynolds, W.C., Schraub, F.A. \& Runstadler, P.W. 1967 The structure of turbulent boundary layers. J.Fluid Mech. 30, 741-773. 